\title{
Blind Beamforming on a Randomly Distributed Sensor Array System
}

\author{
Kung Yao, Fellow, IEEE, Ralph E. Hudson, Chris W. Reed, Daching Chen, and Flavio Lorenzelli
}

\begin{abstract}
We consider a digital signal processing sensor array system, based on randomly distributed sensor nodes, for surveillance and source localization applications. In most array processing the sensor array geometry is fixed and known and the steering array vector/manifold information is used in beamformation. In this system, array calibration may be impractical due to unknown placement and orientation of the sensors with unknown frequency/spatial responses. This paper proposes a blind beamforming technique, using only the measured sensor data, to form either a sample data or a sample correlation matrix. The maximum power collection criterion is used to obtain array weights from the dominant eigenvector associated with the largest eigenvalue of a matrix eigenvalue problem. Theoretical justification of this approach uses a generalization of Szegö's theory of the asymptotic distribution of eigenvalues of the Toeplitz form. An efficient blind beamforming time delay estimate of the dominant source is proposed. Source localization based on a least squares (LS) method for time delay estimation is also given. Results based on analysis, simulation, and measured acoustical sensor data show the effectiveness of this beamforming technique for signal enhancement and space-time filtering.
\end{abstract}

Index Terms - Array processing, beamformer, micro-electromechanical (MEM) sensor, sensor network, source localization, space-time filtering.

\section{INTRODUCTION}

I $\mathrm{N}$ the last 20 years, there has been much interest in the theoretical and practical aspects of beamforming. Beamforming has been used in radar, sonar, and wireless radio applications. Depending on the application, the sources may be narrowband or broadband in the far-field or near-field, there may be multipath or reverberant echoes, and the frequency and spatial responses of the sensors may be completely or partially unknown.

The problem of beamforming in a narrowband environment, with precisely known sensor locations and responses, is well documented. Different algorithms exploit the structure of the steering matrix to obtain information regarding source direction of arrival [1]. Many high-resolution direction of arrival estimation algorithms have been proposed in recent years, but none of them are suited to uncalibrated randomly distributed sensors arrays, passively receiving wideband signals. MUSIC [2] and ESPRIT [3] require narrowband signals. A considerable amount of research has been devoted to the difficult problems of beamforming in the presence of coherent sources

Manuscript received October 1, 1997; revised February 1, 1998. This work was supported in part by the DARPA under TTO Contract F04701-97-C-0010, by NASA under Grant NCC 2-374, and by a Raytheon Ph.D. Fellowship.

The authors are with the Electrical Engineering Department, University of California, Los Angeles, CA 90095-1594 USA.

Publisher Item Identifier S 0733-8716(98)07884-6.
[4] and the effects of imprecisions in the sensor calibration and location have also been investigated [5]. Beamforming for broadband sources has usually been considered as an extension of narrowband beamforming in the frequency domain by use of subband filtering and/or focusing matrix techniques [6]. This method also requires sensor response calibration.

When the information regarding sensor placement and response is partially or totally lacking, the beamforming problem is usually referred to as blind beamforming. Numerous articles have dealt with this topic, usually in the area of digital communications. The typical scenario involves narrowband sources of which some known characteristics are used for the purpose of detection or signal copy. Among the features exploited are: the cyclostationarity property [7]; spectral self-coherence [8] or the finite alphabet property of digital communication signals; the constant modulus characteristic of frequency modulation/phase modulation (FM/PM) signals [9], [10]; the statistical difference between desired and undesired sources, including types of signal nonstationarity [11]; and higher order statistical parameters. The latter class of problems has generated a wide variety of articles in which higher order cumulants have been effectively used to combat the effect of mesokurtic disturbances, such as Gaussian noise [12].

There has been much recent interest in using low-power and low-cost complementary metal-oxide-semiconductor (CMOS) fabricated micro-electro-mechanical (MEM) sensors, in conjunction with modern digital signal processors (DSP's) and radio frequency ( $\mathrm{RF}$ ) radio communication techniques, to tackle various challenging problems involving the coupling of data from the physical world through a network to the end user. In this paper, we consider an array system in which the sensor nodes may be randomly distributed. The user may have the control of some general parameters of placement of these nodes, such as the approximate density of the nodes, and an approximate one-dimensional pattern versus a two-dimensional area deployment. The exact placement, control of orientation, and knowledge of frequency/spatial responses of the sensors, however, are generally considered to be unrealistic. These nodes may contain acoustical, vibrational, and other MEMsensing elements. These nodes, upon sensing an event of interest, may self-organize into a synchronized wireless radio network using low-power spread spectrum transceivers to communicate among themselves and central processors. Data from these nodes may be used to perform various cooperative signal processing and beamforming operations for detection reconfirmation; to reduce the probability of false alarm; source localization, and signal-to-noise ratio (SNR) enhancement for source signature identification, etc. These systems may be 
used in the sensing of information from a modern industrial plant for robotics control; for improving manufacturing efficiency; for home/industrial security; and battlefield intrusion, reconnaissance, and surveillance applications.

In Section II, we first elaborate on the concept of beamforming for an array of randomly distributed sensors which we introduced earlier [13]. The correlation matrix formed from the received data vectors of the sensors is used to perform maximum power collection from the source with the highest peak power spectral density. This operation is based on the use of the array-weighting vectors obtained from the dominant eigenvector, associated with the largest eigenvalue obtained from the matrix eigenvalue problem of the correlation matrix. We also show the ensemble averaged correlation matrix approach, which has a version using the sample data matrix formulation. In Section III, we show that, for broadband sources modeled by wide-sense stationary random processes, the Szegö theory of asymptotic distribution of eigenvalues of the Toeplitz form [16] can be generalized to this arrayprocessing problem to explain the maximum power collecting array. Specifically, the relative phase information among the weights of the array yield the relative propagation time delays from the dominant source of interest to the array sensors. In Section IV, blind beamforming time delay estimations and applications to source localization are presented. Furthermore, the transfer function of the array, using the eigenvector generated weights, forms a narrow bandpass filter centered about the frequency of the maximum peak source spectral density. Performance of various ideal and practical array systems show the effectiveness of the beamforming technique for signal enhancement and space-time filtering. Section V considers a general source localization scheme, based on a least squares (LS) time delay estimation. In Section VI, a brief conclusion and some discussion of ongoing work are given.

\section{MaXimum Power Collecting Array}

Consider the situation in which the sensors are randomly distributed in a spatial region, which can be one, two, or three dimensional. The sensors' relative positions and frequency/spatial responses are unknown for beamformation. The sources may be narrowband or broadband and they may be in the far- or near-field with respect to the sensor array. Reverberation and echoes may exist. The sources do not have specific characteristics that can be used to our advantage. In this paper, we use the term blind beamforming to denote array processing possessing all the conditions characterized above. Others may use blind beamforming when only some of these conditions are imposed. Due to these restrictions, our initial goal is limited to the detection, enhancement, and relative time delay estimation of the strongest signal in the presence of the white noise. In particular, if an initial weaker interfering signal later becomes stronger than the initial strongest desired signal, then our proposed blind beamformer considers this interfering signal as the desired signal and concentrates the beamforming effort toward it. When the locations of the sensors are known, the relative time delay estimates can be used to locate the strongest signal relative to the sensors.
We assume wavefronts from $D$ sources $s_{d}(t), d=$ $1, \cdots, D$ are generated. These wavefronts impinge on an array composed of $R$ sensor elements. The input waveform at the $r$ th sensor $r=1, \cdots, R$ is denoted by

$$
x_{r}(t)=\sum_{d=1}^{D} s_{d}\left(t-t_{d, r}\right)+n_{r}(t)
$$

where $t_{d, r}$ is the propagation time from the $d$ th source to the $r$ th sensor and $n_{r}(t)$ is a temporally and spatially white noise with zero mean and variance $\sigma^{2}$. Consider the simple scenario given in Fig. 1 with $D=2$ sources, $R=3$ sensors, and $L$ taps per sensor channel. Initially, suppose only the wavefronts of $d=1$ drawn in solid circles impact the sensors. Later, we will consider the impact of the $d=2$ wavefronts drawn in dashed circles. Let the sensor input waveform be sampled at the rate of $1 / T$ samples per second. For simplicity of notation, but without losing generality, we set $T=1$ and assume sensor one is the furthest away, followed by sensor two, with sensor three being the closest to source one in Fig. 1. Denote the $L \times 1$ sensor data vector at the three sensors and their combined $3 L \times 1$ sampled vector by

$$
\begin{aligned}
\mathbf{x}_{1} & =[x(n), x(n-1), \cdots, x(n-L+1)]^{T} \\
\mathbf{x}_{2} & =[x(n-p), x(n-p-1), \cdots, x(n-p-L+1)]^{T} \\
\mathbf{x}_{3} & =[x(n-q), x(n-q-1), \cdots, x(n-q-L+1)]^{T} \\
\mathbf{x} & =\left[\mathbf{x}_{1}^{T}, \mathbf{x}_{2}^{T}, \mathbf{x}_{3}^{T}\right]^{T}
\end{aligned}
$$

where $p=t_{12}$ is the relative time delay of the first sensor to the second sensor, $q=t_{13}$ is the relative time delay of the first sensor to the third sensor, and $T$ is the transpose operator. From the earlier assumption on the sensor distances to source one and the assumption that the array response is longer than the largest of the relative time delays, we have $0<p<q<L-1$. Denote the auto and crosscorrelation matrices of $\mathrm{x}_{1}$ with $\mathrm{x}_{1}, \mathrm{x}_{1}$ with $\mathrm{x}_{2}, \mathrm{x}_{1}$ with $\mathrm{x}_{3}, \mathrm{x}_{2}$ with $\mathrm{x}_{3}$, and $\mathrm{x}$ with $\mathrm{x}$, respectively, as

$$
\begin{aligned}
& \mathbf{R}_{L}^{11}=E\left\{\mathbf{x}_{1} \mathbf{x}_{1}^{H}\right\}=E\left\{\mathrm{x}_{2} \mathbf{x}_{2}^{H}\right\}=E\left\{\mathbf{x}_{3} \mathbf{x}_{3}^{H}\right\} \\
& \mathbf{R}_{L}^{12}=E\left\{\mathbf{x}_{1} \mathbf{x}_{2}^{H}\right\} \\
& \mathbf{R}_{L}^{13}=E\left\{\mathbf{x}_{1} \mathbf{x}_{3}^{H}\right\} \\
& \mathbf{R}_{L}^{23}=E\left\{\mathbf{x}_{2} \mathbf{x}_{3}^{H}\right\} \\
& \mathbf{R}_{3 L}=E\left\{\mathbf{x x}^{H}\right\}=\left[\begin{array}{lll}
\mathbf{R}_{L}^{11} & \mathbf{R}_{L}^{12} & \mathbf{R}_{L}^{13} \\
\mathbf{R}_{L}^{12} & \mathbf{R}_{L}^{11} & \mathbf{R}_{L}^{23} \\
\mathbf{R}_{L}^{13} \mathbf{R}_{L}^{23 H} & \mathbf{R}_{L}^{11}
\end{array}\right]
\end{aligned}
$$

where $*$ is the complex conjugation and $H$ is the complex conjugate transpose. Since the noise is temporally and spatially white, the component of $\mathbf{R}_{3 L}$ due to the noise is simply $\sigma^{2} \mathbf{I}$.

In general, we want to find an algorithm that generates the beamformer output

$$
y(n)=\sum_{r=1}^{R} \sum_{\ell=0}^{L-1} w_{r \ell}^{*} x_{r}(n-\ell)
$$

to satisfy some desired criterion. In (3), $w_{r \ell}$ denotes the $\ell$ th array-weight coefficient of the $r$ th sensor. The combined $3 L \times 1$ 


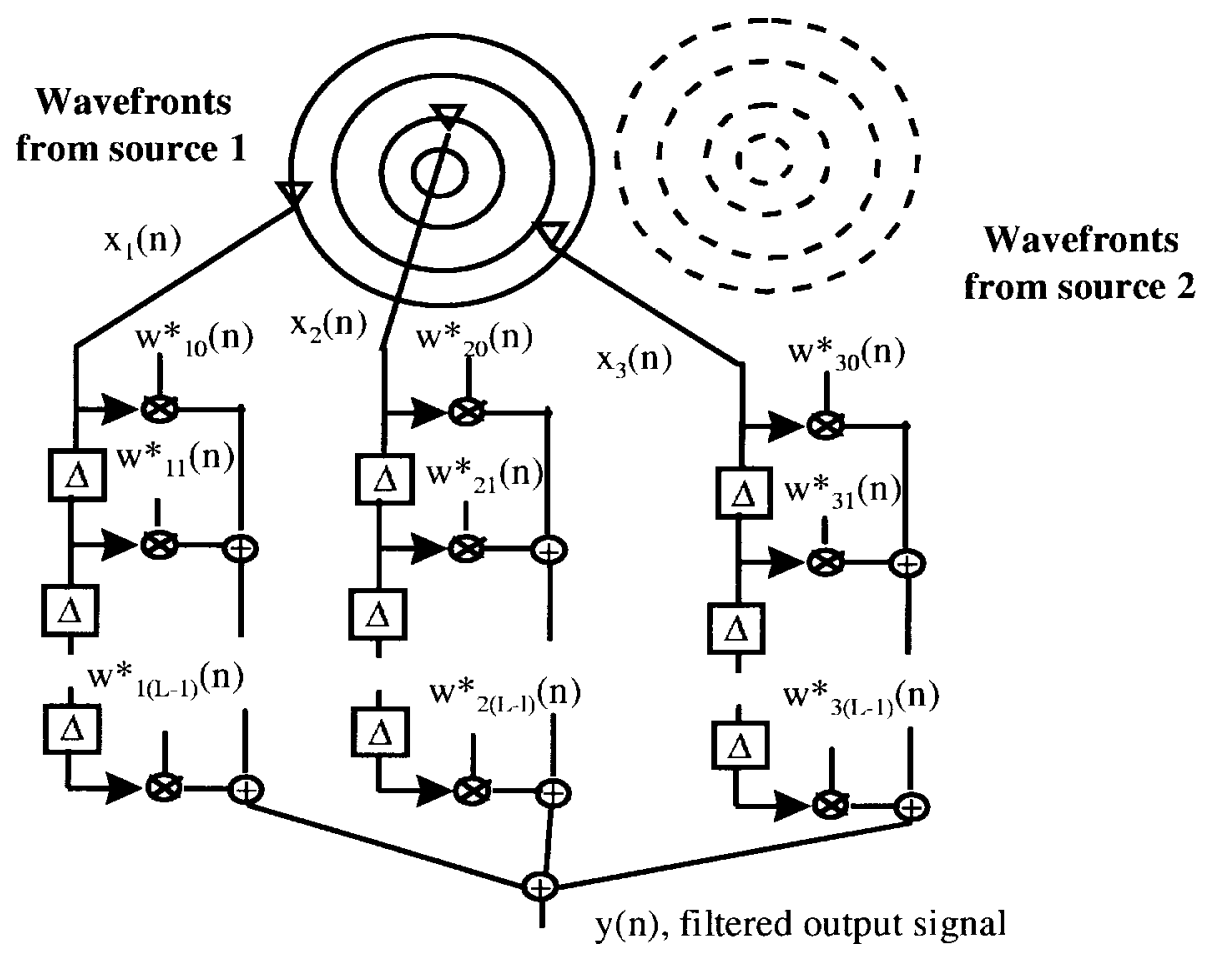

Fig. 1. Beamforming with three randomly distributed sensors and $L$ taps.

array-weight vector is denoted by

$$
\begin{gathered}
\mathbf{w}_{3 L}=\left[w_{10}, w_{11}, \cdots, w_{1(L-1)}, w_{20}, \cdots, w_{2(L-1)}, \cdots,\right. \\
\left.w_{30}, \cdots, w_{3(L-1)}\right]^{T} .
\end{gathered}
$$

Now, assume the objective of the sensor array is to detect the presence of the strongest source which emits the signal $s_{1}(t)$ in an otherwise relatively quiet environment. The sensor array is required to pick up the signal, even possibly a distorted version of it, while rejecting other sources of disturbance, such as interferers and noise. Assuming that $s_{1}(t)$ is the strongest signal received by the sensors, then one can choose the weights in such a way as to maximize the output power constrained to $\left\|\mathbf{w}_{3 L}\right\|^{2}=\sum_{r} \sum_{\ell}\left|w_{r \ell}\right|^{2}=1$. The array output is a linear combination of delayed versions of the impinging signals. It is reasonable to expect that the combination which corresponds to the largest output power is the one that sums coherently the strongest of the signals, to the disadvantage of the weaker signals. The solution to this objective is obtained by solving the following maximization problem:

$$
\text { maximize }\left\{\mathbf{w}_{3 L}^{H} \mathbf{R}_{3 L} \mathbf{w}_{3 L}\right\}, \quad \text { subject to }\left\|\mathbf{w}_{3 L}\right\|=1
$$

where $\mathbf{R}_{3 L}$ is the space-time correlation matrix of (2). The unity constraint on the norm of the weights $\mathbf{w}_{3 L}$ ensures that the array output noise power is the same as the input noise power. The maximization in (5) is equivalent to maximizing the SNR at the output of the array. The desired $\mathbf{w}_{3 L}$ is then given by the $3 L \times 1$ eigenvector $\phi_{3 L}^{(3 L)}$, corresponding to the largest eigenvalue of $\mathbf{R}_{3 L}$, in the following matrix eigenvalue problem:

$$
\begin{aligned}
\mathbf{R}_{3 L} \phi_{k}^{(3 L)}= & \lambda_{k}^{(3 L)} \phi_{k}^{(3 L)} \\
& 0 \leq \lambda_{1}^{(3 L)} \leq \cdots \leq \lambda_{k}^{(3 L)} \leq \cdots \leq \lambda_{3 L}^{(3 L)} \\
& 1 \leq \cdots \leq k \leq \cdots \leq 3 L
\end{aligned}
$$

The auto and crosscorrelation matrices $\mathbf{R}_{L}^{i j}$ and $\mathbf{R}_{3 L}$ in (2) use ensemble averaging on the random vectors in (1). Now, we present a time-averaged sample correlation matrix formulation. Then the array weights are obtained from the dominant eigenvector of this time-averaged sample correlation matrix. Denote the received signal at sensor $r$ by $x_{r}(n)$ where $r=1,2,3$. The $L \times 1$ sampled sensor data vector at sensor $r$, taken at the $k$ th snapshot, can be written as $\mathbf{x}_{r}(k)=\left[\mathbf{x}_{r}(n+k), \mathbf{x}_{r}(n+k-1), \cdots, \mathbf{x}_{r}(n+k-L+1)\right]^{T}$ and the combined $3 L \times 1$ sampled vector can be written as $\mathbf{x}(k)=\left[\mathbf{x}_{1}(k)^{T}, \mathbf{x}_{2}(k)^{T}, \mathbf{x}_{3}(k)^{T}\right]^{T}$. Let $k=0,1, \cdots, N-1$ denote the $N$ snapshots available for computations. The total number of samples in these snapshots at each sensor is thus $L+N-1$. Using these $N$ snapshots, or an equivalent $L+N-1$ samples at each sensor, an approximation of the time-average sample correlation matrix $\mathbf{R}_{3 L}$ in (2) can be given by

$$
\hat{\mathbf{R}}_{3 L}=\frac{1}{N} \sum_{k=0}^{N-1} \mathbf{x}(k) \mathbf{x}(k)^{H}=\left[\begin{array}{ccc}
\hat{\mathbf{R}}_{L}^{11} & \hat{\mathbf{R}}_{L}^{12} & \hat{\mathbf{R}}_{L}^{13} \\
\hat{\mathbf{R}}_{L}^{21} & \hat{\mathbf{R}}_{L}^{22} & \hat{\mathbf{R}}_{L}^{23} \\
\hat{\mathbf{R}}_{L}^{31} & \hat{\mathbf{R}}_{L}^{32} & \hat{\mathbf{R}}_{L}^{33}
\end{array}\right]
$$

where $\hat{\mathbf{R}}_{L}^{r s}=(1 / N) \sum_{k=0}^{N-1} \mathbf{x}_{r}(k) \mathbf{x}_{s}(k)^{H}, r, s=1,2,3$. Denote the three $L \times N$ data matrices $\mathbf{A}_{r}^{H}, r=1,2,3$ as shown in (7a) at the bottom of the next page.

We can form a $3 L \times N$ data matrix $\mathbf{A}^{H}$ from $\mathbf{A}_{1}^{H}, \mathbf{A}_{2}^{H}$, and $\mathbf{A}_{3}^{H}$ as

$$
\mathbf{A}^{H}=\frac{1}{\sqrt{N}}\left[\begin{array}{l}
\mathbf{A}_{1}^{H} \\
\mathbf{A}_{2}^{H} \\
\mathbf{A}_{3}^{H}
\end{array}\right]=\frac{1}{\sqrt{N}}[\mathbf{x}(0), \mathbf{x}(1), \cdots, \mathbf{x}(N-1)] .
$$

Direct evaluation shows that $\hat{\mathbf{R}}_{3 L}=\mathbf{A}^{H} \mathbf{A}$. Therefore, the $3 L \times 1$ right singular vectors of $\mathbf{A}$ are also the corresponding 
$3 L \times 1$ eigenvectors of $\hat{\mathbf{R}}_{3 L}$, and the singular values of $\mathbf{A}$ are just the square roots of the corresponding eigenvalues of $\hat{\mathbf{R}}_{3 L}$ [14]. The technique considered above on the eigendecomposition of the $3 L \times 3 L$ ensemble averaged space-time correlation matrix $\mathbf{R}_{3 L}$ in (2) applies equally to the time-averaged space-time correlation matrix of (7) for arrayweight evaluation. Similarly, we can apply the singular value decomposition [15] directly on the $3 L \times N$ data matrix $\mathbf{A}$ of (8) and find the $3 L \times 1$ right singular vector corresponding to the largest singular value for array-weight evaluation. Under ideal infinite precision computations, the eigenvector and singular vector solutions of (7) and (8) are equivalent. The computational cost of evaluating the dominant singular vector, however, may be higher than the cost of evaluating the dominant eigenvector using the power method [15]. In the next section, some detailed properties of the maximum power collection of the array discussed here are derived, based on the Szegö theory of asymptotic distribution of eigenvalues of a Toeplitz form applied to a wide-sense stationary random sequence.

\section{MaXimum Power Collection AND SZegö's Method}

Consider a wide-sense stationary random process $\{x(t),-\infty<t<\infty\}$. Upon sampling $x(t)$ every $T$ second, the sampled wide-sense stationary random sequence is denoted by $\{x(n),-\infty<n<\infty\}$. Without loss of generality, we can set $T=1$. Let $r(n)=E\left\{x(n+m) x(m)^{*}\right\}$ denote the autocorrelation function of $\{x(n),-\infty<n<\infty\}$. Then, by the Bochner Theorem, the power spectral density function $S(f)$ is related to $\{r(n),-\infty<n<\infty\}$ by $S(f)=\sum_{n=-\infty}^{\infty} r(n) e^{-i 2 \pi f n},-0.5 \leq f<0.5$ where $r(n)=\int_{-0.5}^{0.5} S(f) e^{i 2 \pi f n} d f,-\infty<n<\infty$.

From the classical Szegö theory of asymptotic distribution of eigenvalues for a wide-sense stationary random sequence [16] we have

$$
\int_{-0.5}^{0.5} S(f) d f=\lim _{L \rightarrow \infty} \frac{\lambda_{1}^{(L)}+\cdots+\lambda_{L}^{(L)}}{L}
$$

where $\lambda_{k}^{(L)}$ is the $k$ th eigenvalue of the matrix eigenvalue problem

$$
\begin{gathered}
\mathbf{R}_{L}^{11} \phi_{k}^{(L)}=\lambda_{k}^{(L)} \phi_{k}^{(L)}, 0 \leq \lambda_{1}^{(L)} \leq \cdots \lambda_{k}^{(L)} \cdots \leq \lambda_{L}^{(L)}, \\
1 \leq \cdots \leq k \leq \cdots \leq L
\end{gathered}
$$

and $\mathbf{R}_{L}^{11}$ is the $L \times L$ correlation matrix of $\mathbf{x}_{1}$ with $\mathbf{x}_{1}$ defined in (1). We note $\mathbf{R}_{L}^{11}=\left[r_{m, n}\right]$ is a Toeplitz matrix since its $(m, n)$ th element for $1 \leq m, n \leq L$ satisfies the Toeplitz condition of $r_{m, n}=r(n-m)$. This means that all the elements along all the diagonals are identical. A generalization [16] of the result in (9) states that for any continuous function

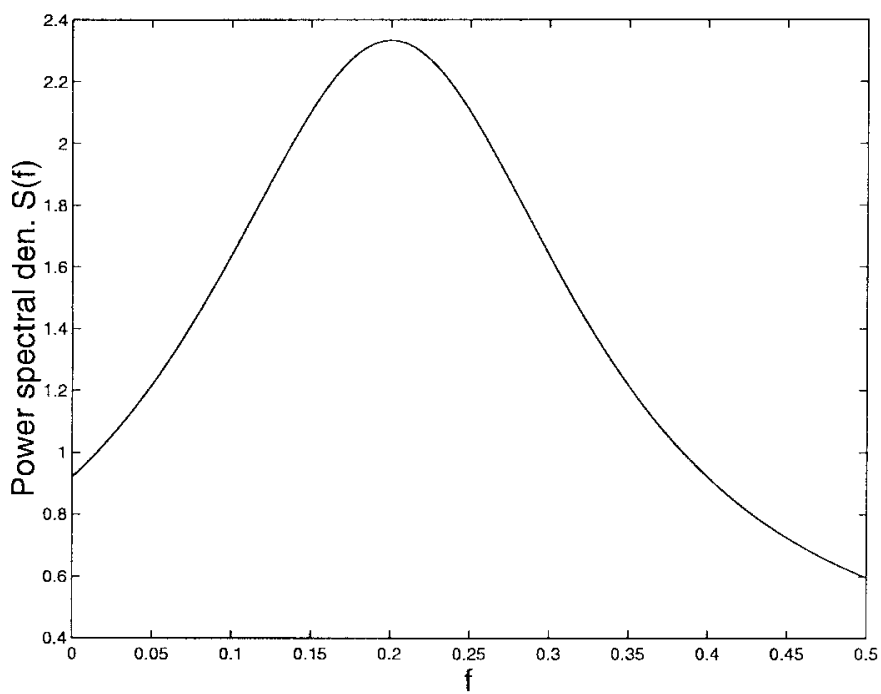

Fig. 2. First-order AR power spectral density versus frequency $f$.

$g(\cdot)$ defined over $[\min \{S(f)\}, \max \{S(f)\}]$

$$
\int_{-0.5}^{0.5} g(S(f)) d f=\lim _{L \rightarrow \infty} \frac{g\left(\lambda_{1}^{(L)}\right)+\cdots+g\left(\lambda_{L}^{(L)}\right)}{L} .
$$

In particular, by taking $g(\cdot)=\max (\cdot)$, then the result in (9) becomes

$$
\begin{aligned}
S_{\max } & =\int_{-0.5}^{0.5} \max (S(f)) d f=\lim _{L \rightarrow \infty} \lambda_{L}^{(L)} \approx \lambda_{L}^{(L)} \\
& =\max _{\left\|\mathbf{w}_{L}\right\|=1}\left\{\mathbf{w}_{L}^{H} \mathbf{R}_{L} \mathbf{w}_{L}\right\}, \text { for sufficiently large } L .
\end{aligned}
$$

We note the result in (11) is crucially used in the formal derivation of the Shannon water-filling colored Gaussian noise channel capacity theorem [17]. For the array processing power maximization application, we need to determine when a finite but large $L$ makes the right-hand side a good approximation to the asymptotic result on the left-hand side of (11). We consider some specific cases to illustrate this issue.

Case 1: Consider a first-order autoregressive (AR) random sequence with $a_{1}=0.4 e^{i 2 \pi 0.2}$. Then its autocorrelation function is given by $r(n)=a_{1}^{n}, n \geq 0 ; r(n)=a_{1}^{*(-n)}, n<0$ and its power spectral density is given by $S(f)=(1-$ $\left.\left|a_{1}\right|^{2}\right) /\left(\left|e^{i 2 \pi f}-a_{1}\right|^{2}\right), 0 \leq f \leq 0.5$. A plot of $S(f)$ is given in Fig. 2 and it has a peak power spectral density of $S_{\max }=2.3333$. A plot of the estimated power spectral density peak $\lambda_{L}^{(L)}$, versus $L$ evaluated from (11), is given in Fig. 3 . For this case, the asymptotic result is achieved for values of $L$ greater than approximately 50 .

Case 2: Consider how the wavefronts from a source with the same parameters as those considered in Case 1 impact on the three sensors, as described in Fig. 1. Let the relative

$$
\mathbf{A}_{r}^{H}=\frac{1}{\sqrt{N}}\left[\mathrm{x}_{r}(0), \cdots, \mathbf{x}_{r}(N-1)\right]=\frac{1}{\sqrt{N}}\left[\begin{array}{cccc}
x_{r}(n) & x_{r}(n+1) & \cdots & x_{r}(n+N-1) \\
x_{r}(n-1) & x_{r}(n) & \cdots & x_{r}(n+N-2) \\
\vdots & \vdots & \ddots & \vdots \\
x_{r}(n-L+1) & x_{r}(n-L+2) & \cdots & x_{r}(n+N-L)
\end{array}\right]
$$




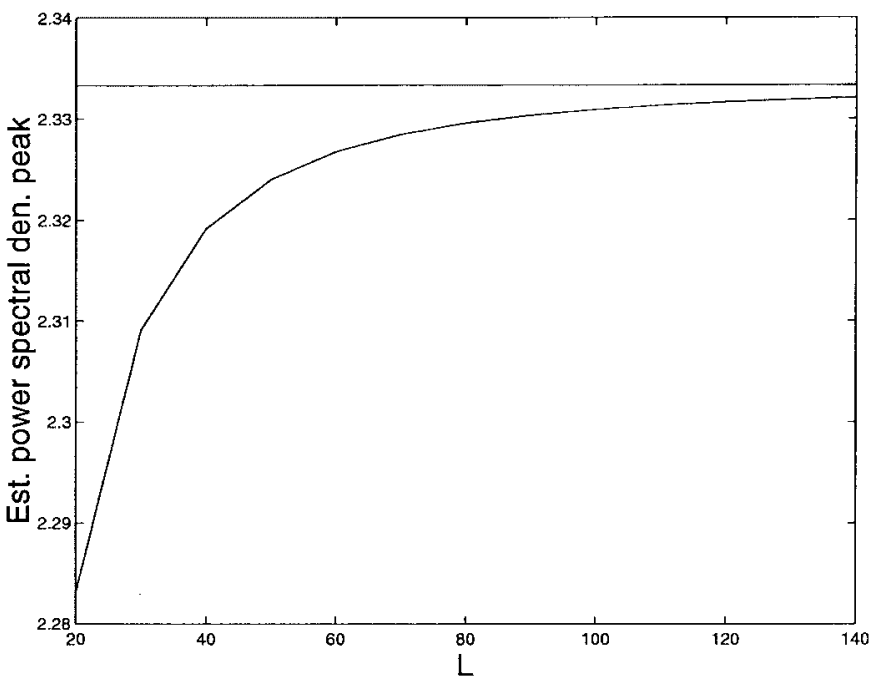

Fig. 3. Estimated power spectral density peak values versus $L$ taps.

time delay $p=t_{12}=3$ and $q=t_{13}=5$. Then the $3 L \times 3 L$ correlation matrix $\mathbf{R}_{3 L}$ has the form as given in (2). We note that $\boldsymbol{R}_{3 L}$ is no longer a Toeplitz matrix since, in general, elements above the main diagonal are not equal (i.e., $\mathbf{R}_{L}^{12} \neq \mathbf{R}_{L}^{13}$ ). We note, however, that each of the nine submatrices in (2) is still a Toeplitz matrix. Thus, $\mathbf{R}_{3 L}$ is called a block-Toeplitz matrix. The crucial question is whether the Szegö asymptotic distribution of eigenvalues technique for a Toeplitz matrix can be generalized to that of a blockToeplitz matrix. Recent work by Koga and Cheng [18] and Voois [19] showed that the classical Szegö result can be generalized to a Toeplitz block-Toeplitz matrix, where not only are all the blocks Toeplitz (as in our $\mathbf{R}_{3 L}$ ), but all the block submatrices along any diagonals are also identical. Fortunately, the asymptotic equivalence technique used in the classical Toeplitz matrix that can be extended to the Toeplitz block-Toeplitz, can also be extended to our block-Toeplitz correlation matrix $\mathbf{R}_{3 L}$. Details on a formal proof of this claim will not be presented here, but some intuitive justification and numerical results confirming this result will be presented.

Fig. 4 shows some finite sections of an infinite-dimensional Toeplitz matrix. First, we note that the autocorrelation matrix $\mathbf{R}_{L}^{11}$ is an $L \times L$ section cut from this infinite-dimensional matrix. It has $r(0)$ along its diagonal and is Hermitian. However, each of the crosscorrelation matrices $\mathbf{R}_{L}^{12}, \mathbf{R}_{L}^{13}$, and $\mathbf{R}_{L}^{23}$ is not Hermitian and can be considered to be an $L \times L$ section translated $p, q$, and $(q-p)$ positions, respectively, to the right from the original $\mathbf{R}_{L}^{11}$ in this infinite-dimensional matrix. We also note that, for fixed $p$ and $p$ values, the finite number of rows in these three crosscorrelation matrices that are different from $\mathbf{R}_{L}^{11}$ become less relevant as $L$ increases to infinity. Numerically, we want to know for what values of $L$ is the generalized asymptotic distribution of eigenvalues valid. Ideally, the total power spectral density of the three combined sensors should be three times that of a single sensor (in the absence of noise). Thus, we expect $S_{\max }=3 \times 2.3333=7$. The estimated total power spectral density peak $\lambda_{3 L}^{(3 L)}$, by the generalized Szegö technique, is plotted as a function of $L$ in Fig. 5. We note a value of $L \geq 60$ converges quite rapidly to the asymptotic value. From our experience with various cases, quite modest values of $L$ can approximate the asymptotic result well.

\section{Blind BeAmforming Time Delay ESTIMATION AND SOURCE LOCALIZATION}

In this section, we consider the use of blind beamforming to estimate the time delays from the dominant source, with and without interference, to the sensors. From the eigenvector $\phi_{3 L}^{3 L}$ corresponding to the largest eigenvalue $\lambda_{3 L}^{(3 L)}$ of (6), define the array weight of length $L$ by $\mathbf{w}_{L}^{(r)}=\phi_{3 L}^{3 L}(r L$ : $-1:(r-1) L+1), r=1,2,3$. From Section II, we could have stated this result equally well by using the $3 L \times 1$ dominant eigenvector or singular vector, computed from the time-averaged sample correlation matrix or direct data matrix. From Section III, in order to achieve the largest possible power at the output of the array in (3), the beamformer chooses, in the spatial and frequency domains, the source with the largest peak spectral density value. This means the beamformer must align the data in each channel properly in phase and forms a narrow band filter at the frequency of the peak spectral value. We can obtain much insight on these signal enhancement and frequency and spatial separation issues by examining the previously used simple AR source and a measured tracked vehicle acoustic-sensor source.

Case 3: We use the AR source in Case 1 with three sensors with relative time delays of $p=t_{12}=3$ and $q=t_{13}=5$, as in Case 2. While these delays are all integral values, the proposed beamforming method is equally applicable for nonintegral delay values. Fig. 6 shows the plot of the magnitudes of the three array weights. We note these weights are just delayed versions of each other. Table I shows the first 15 values of $\left|\mathbf{w}_{L}^{(1)}\right|,\left|\mathbf{w}_{L}^{(2)}\right|$, and $\left|\mathbf{w}_{L}^{(3)}\right|$. We note that the values of $\left|\mathbf{w}_{L}^{(2)}\right|$ are three time units behind that of $\left|\mathbf{w}_{L}^{(1)}\right|$, while $\left|\mathbf{w}_{L}^{(3)}\right|$ is five time units behind that of $\left|\mathbf{w}_{L}^{(1)}\right|$. This is consistent with their known relative time delays. Fig. 7 shows the magnitude of the transfer function of the first array filter obtained from the magnitude of the fast Fourier transform (FFT) of $\mathbf{w}_{L}^{(1)}$. The result is identical if we use the magnitude of the FFT of the other two weights. Thus, each of the three array finite impulse response (FIR) filters acts as a narrow bandpass filter centered at the frequency $f_{\max }=0.2$, which yields the maximum source spectral density value. For this broadband source, the wavefronts received at the second sensor are delayed by two time units, while the wavefronts received at the third sensor are delayed by five time units, all relative to the first sensor, and thus add coherently at the output of the beamformer. In the absence of noise, the output power is three times larger than that of a single channel output.

Case 4: Consider a measured tracked vehicle acoustic source [20] with a spectral peak at approximately $100 \mathrm{~Hz}$, plus an interferer modeled by a second-order AR source of coefficients $a_{1}=-2 * 0.989 \cos (2 \pi * 0.12)$ and $a_{2}=0.989^{2}$ resulting in a spectral peak of approximately $120 \mathrm{~Hz}$, as shown in Fig. 8. The $x-y$ coordinates of the three sensors are given by $\{(12,0),(0,12),(-9,0)\}$, the tracked vehicle given by $\{(7,-12)\}$, and the interferer are given by $\{(6.08$, $-8.438)\}$. The true vehicle time delays are 12,7 , and 5 while 


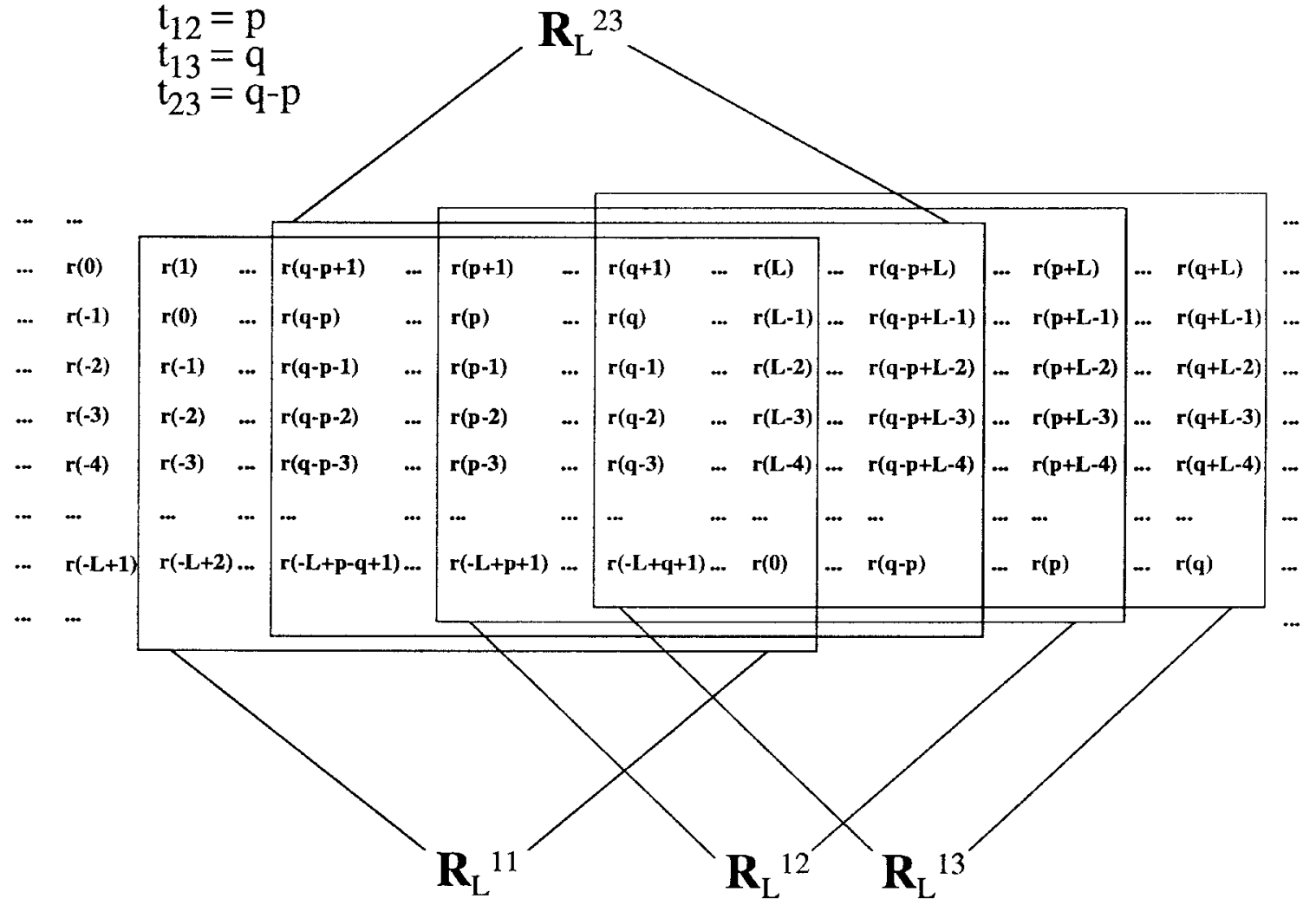

Fig. 4. Relations of $\mathbf{R}_{L}^{12}, \mathbf{R}_{L}^{13}$, and $\mathbf{R}_{L}^{23}$ to $\mathbf{R}_{L}^{11}$ in the infinite-dimensional Toeplitz matrix.

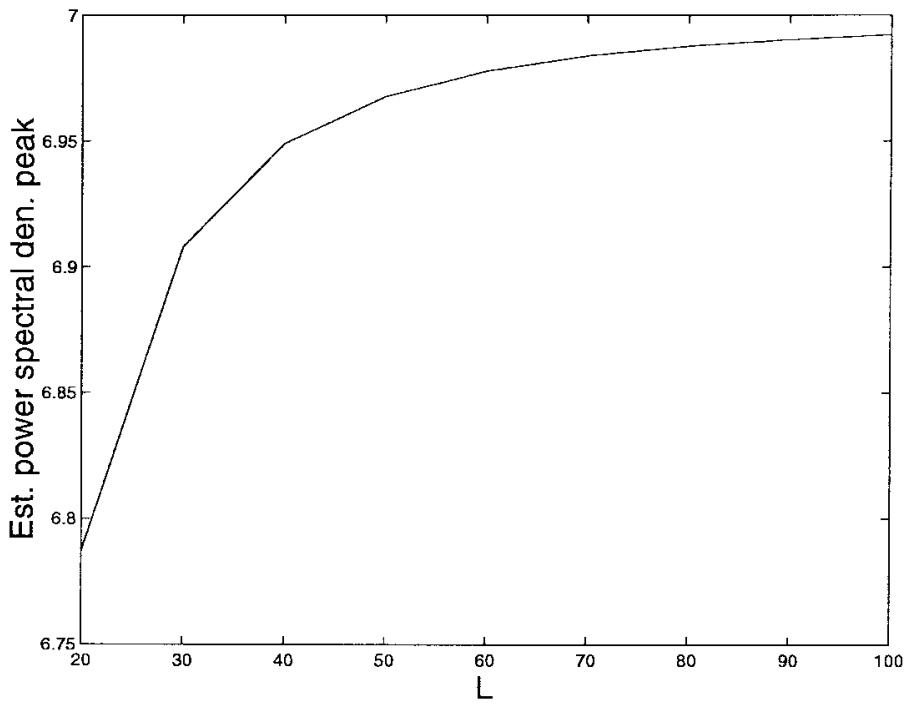

Fig. 5. Estimated spectral density peak values for a three-sensor array versus $L$.

the true interferer time delays are 11,7 , and 4 , all among the sensors. Fig. 9(a) shows the time delay estimates based on our proposed dominant eigenvector method, while Fig. 9(b) shows these estimates based on the classical correlation method operating directly on the sensor data [21]. For a signal-tointerference ratio (SIR) higher than approximately $4 \mathrm{~dB}$, the eigenvector method finds the delays of the strongest source (i.e., the vehicle) with essentially no error. For an SIR of less than approximately $3 \mathrm{~dB}$, the strongest source is the

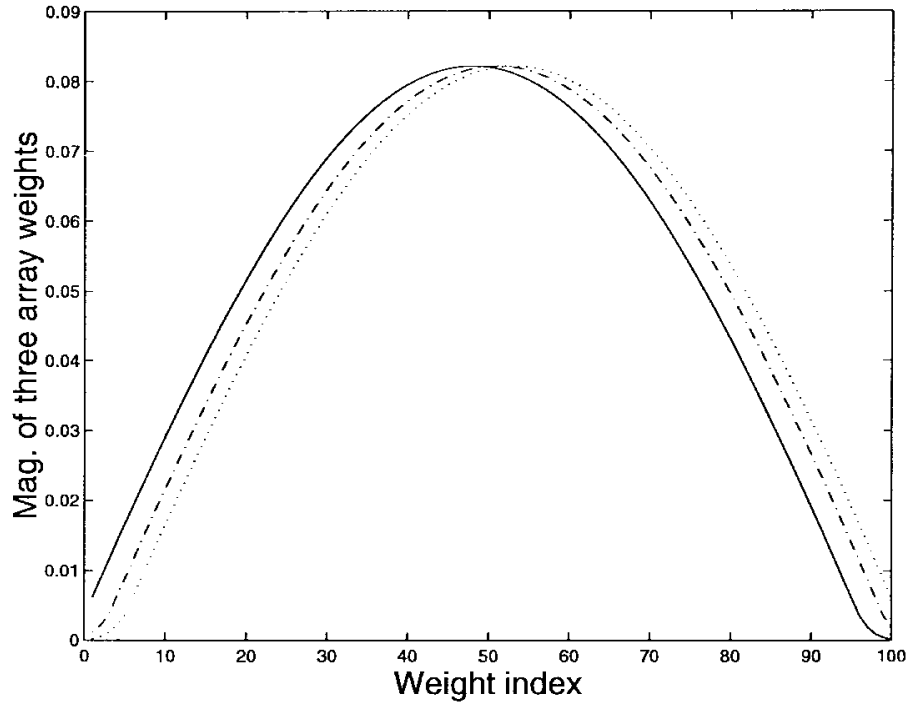

Fig. 6. Magnitude of the three array weights versus weight index.

interferer and its delays are also found with essentially no errors. The estimated delay uncertainty region is only about $1 \mathrm{~dB}$. Fig. 9(b) shows that the classical correlation method yields much less precise estimation results and the estimated delay uncertainty region is about $20 \mathrm{~dB}$. Fig. 10(a) shows the eigenvector-method-estimated $x$ and $y$ coordinates of the dominant source as a function of the SIR, while Fig. 10(b) shows those based on the classical correlation method. The advantage of having a smaller uncertainty interval for the eigenvector method, over the classical correlation method 


\begin{tabular}{cccl}
\multicolumn{5}{c}{ TABLE I } \\
VALUES OF $\left|\mathbf{w}_{L}^{(1)}\right|,\left|\mathbf{w}_{L}^{(2)}\right|$ and $\left|\mathbf{w}_{L}^{(3)}\right|$ \\
\hline $\mathrm{n}$ & $\left|\mathbf{w}_{L}^{(1)}\right|$ & $\left|\mathbf{w}_{L}^{(2)}\right|$ & $\left|\mathbf{w}_{L}^{(3)}\right|$ \\
1 & 0.0062 & 0.0011 & 0.0002 \\
2 & 0.0088 & 0.0020 & 0.0005 \\
3 & 0.0114 & 0.0036 & 0.0011 \\
4 & 0.0140 & 0.0062 & 0.0020 \\
5 & 0.0165 & 0.0088 & 0.0036 \\
6 & 0.0191 & 0.0114 & 0.0062 \\
7 & 0.0216 & 0.0140 & 0.0088 \\
8 & 0.0241 & 0.0165 & 0.0114 \\
9 & 0.0266 & 0.0191 & 0.0140 \\
10 & 0.0291 & 0.0216 & 0.0165 \\
11 & 0.0315 & 0.0241 & 0.0191 \\
12 & 0.0339 & 0.0266 & 0.0216 \\
13 & 0.0363 & 0.0291 & 0.0241 \\
14 & 0.0386 & 0.0315 & 0.0266 \\
15 & 0.0409 & 0.0339 & 0.0291 \\
\hline
\end{tabular}

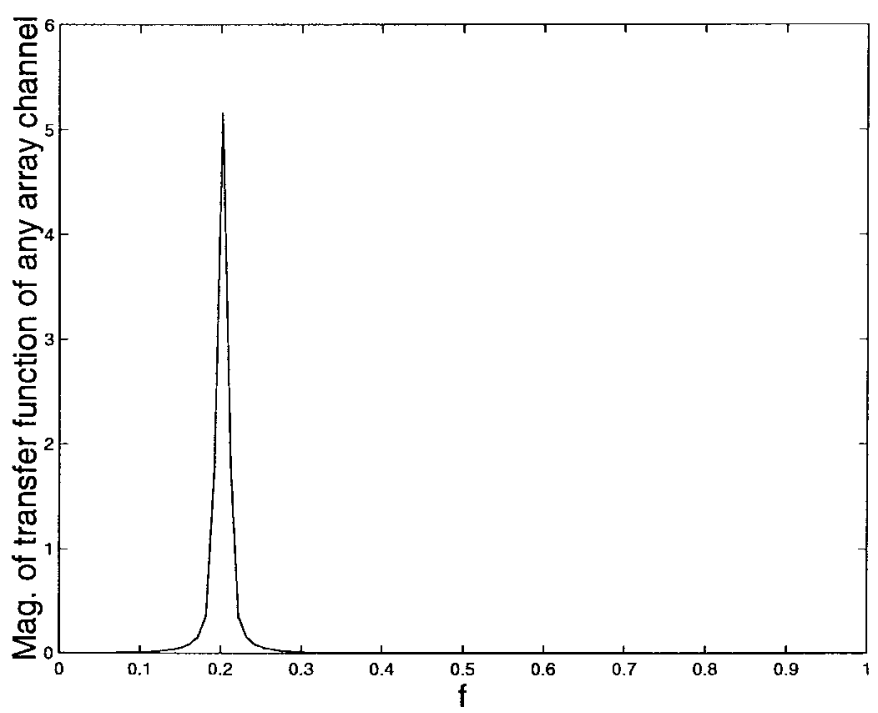

Fig. 7. Magnitude of the transfer function of any array channel versus frequency.

in time delay estimation, is translated directly to a smaller uncertainly in the $x-y$ coordinate estimation. In Fig. 11(a), for the eigenvector method, we see the locations of the three sensors and the tracked vehicle and the interferer. The three dashed curves represent the hyperbolas of constant time delays of $t_{21}=12, t_{31}=7, t_{23}=5$. Furthermore, for high SIR, the eigenvector method estimates the tracked vehicle correctly, so that only a single $x$ mark appears at its true location in Fig. 11(a), while for low SIR, the interferer is estimated correctly and only a single $x$ mark appears at its true location. In Fig. 11(b), however, for the classical correlation method, the estimated dominant source location is estimated continuously between the two true locations as a function of the SIR. We note in this case, the "desired source," the blind beamformer, is concentrating changes from the vehicle to the AR source when the SIR changes from large to small values.
Case 5: We consider the use of the blind beamforming dominant space-time eigenvector method to enhance the SNR of a desired signal in white Gaussian noise (WGN). The signal is taken to be an acoustic tracked vehicle source as was used in Case 4. Ten randomly delayed copies of the signal (with integer delays uniformly distribution from zero to ten) are created to simulate propagation to an array of ten randomly spaced sensors. Each sensor is corrupted by WGN to create an SNR of $-10 \mathrm{~dB}$ per channel. SNRI is defined as the ratio of the beamformer $\mathrm{SNR}_{\text {out }}$ to the $\mathrm{SNR}_{\text {in }}$. Table II shows the SNRI for the blind beamformer, based on the dominant eigenvector method for six different conditions.

In Condition 1, the true time delay beamformer delays each received signal to align them prior to summing them. It assumes the exact delays are known or estimated with high precision. In Conditions 2-4, the eigenvector method weights with 4,10 , and 19 taps are used to obtain significant signal enhancements. In Conditions 5-6, perfect presteering is first used to align the received signals, as in true time delay beamforming, and is followed by the use of eigenvector weights with four and ten taps. We note the SNRI of Conditions 5-6 with presteering are slightly higher than those in corresponding Conditions 2-3, without presteering for an equal number of taps. In practice, there is a tradeoff between a higher/lower SNRI and narrower/broader bandpass filtering, using a larger/smaller number of taps in the beamformer. For some applications a higher SNRI may be of primary interest, while for other situations a flatter frequency response, resulting in a lower distortion, may be more relevant.

Case 6: In Case 4 we demonstrated that if there is sufficient spectral separation between the source and interferer, the eigenvector method can provide reliable estimates of the time delays among the sensors. These time delays are associated with the stronger signal, and the transition range in the SIR is smaller for the eigenvector method than for the conventional crosscorrelation method. Now we use the eigenvector method, which exploits the spatial separation, to also provide these advantages even when there is no spectral separation. The desired source is the same tracked vehicle as in Case 4 and is located spatially at $(7,-12)$, while the interfering source is modeled by a sufficiently delayed (thus independent) version of the desired source, but located spatially at $(0,18)$. The four sensors are located at $(12,0),(0,12),(-9,0)$, and $(7,0)$. Fig. 12(a)-(c) show the relative time delays of $t_{21}, t_{23}, t_{14}$ versus the SIR using the eigenvector method. We note that this method can still estimate correctly the time delays of the source with the peak power spectral density. Fig. 12(d) plots SIRI $=\left(\mathrm{SIR}_{\text {out }} / \mathrm{SIR}_{\text {in }}\right)$ versus $\mathrm{SIR}_{\text {in }}$. These results show the spatial filtering property of our blind beamformer. It is important to note that, for arrays that are quite sparse with separations of many wavelengths between sensors, there may be an existence of spatial ambiguities. Case 6 demonstrates the spatial filtering property of our blind beamforming eigenvector method when the interferer is not located at one of the spatial ambiguities of the desired source. Obviously, our proposed approach, and most other approaches, have limitations if there is a small difference in both the frequency and spatial responses of the sources. 

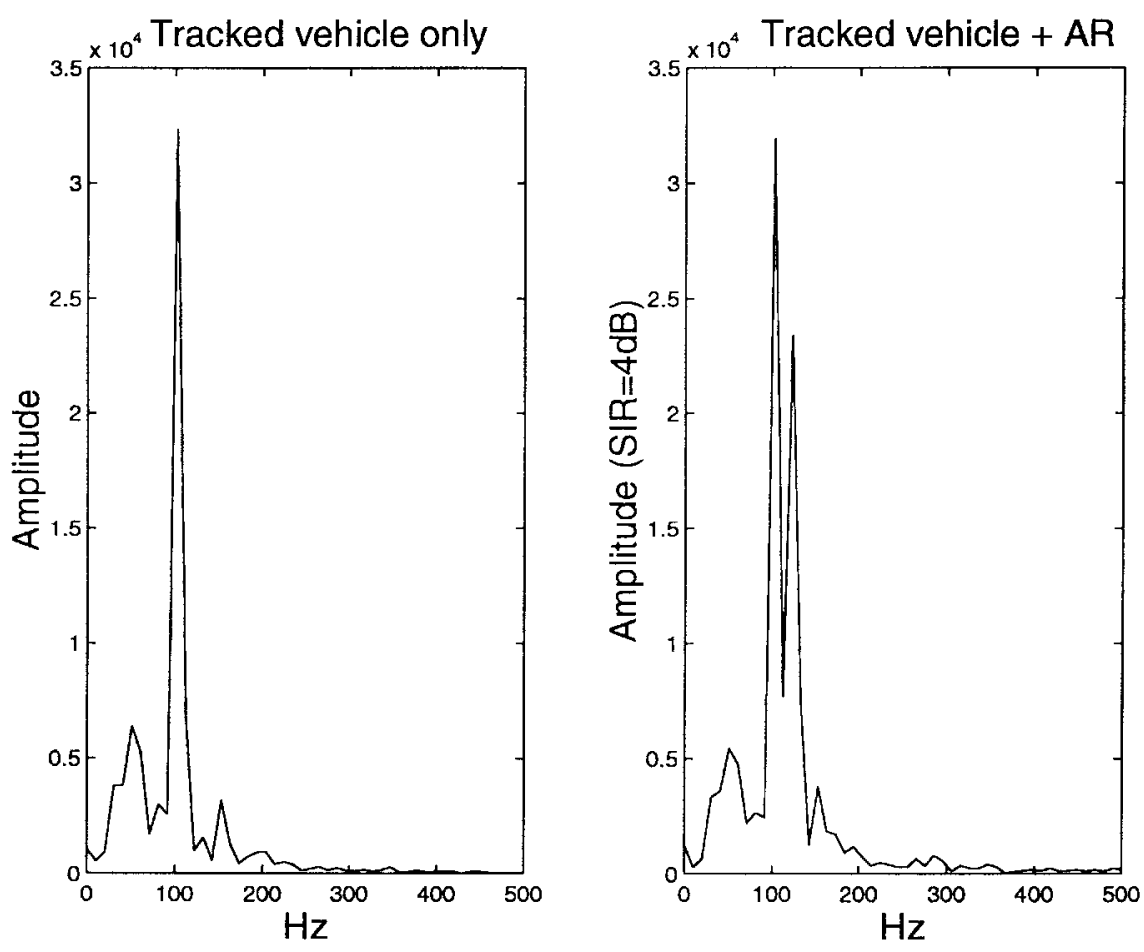

Fig. 8. Amplitudes of tracked vehicle and AR sources.

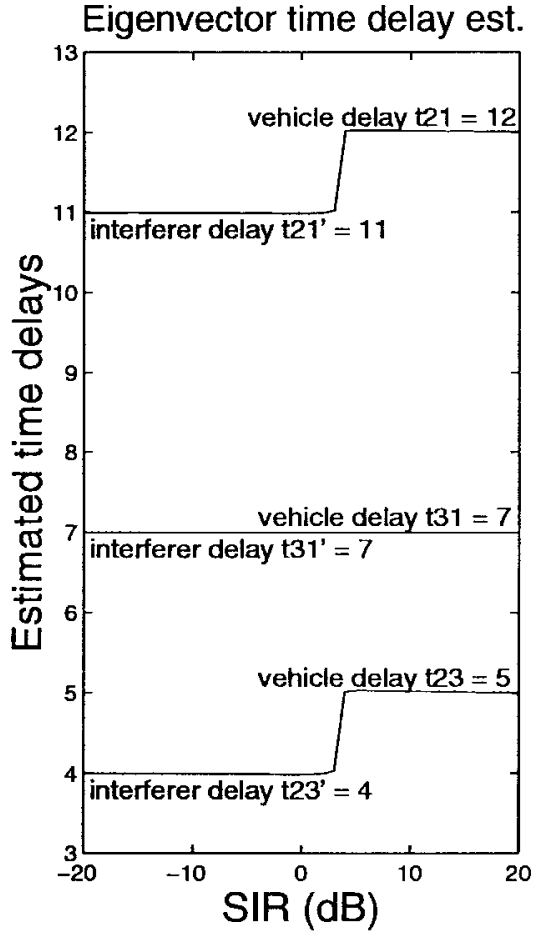

(a)

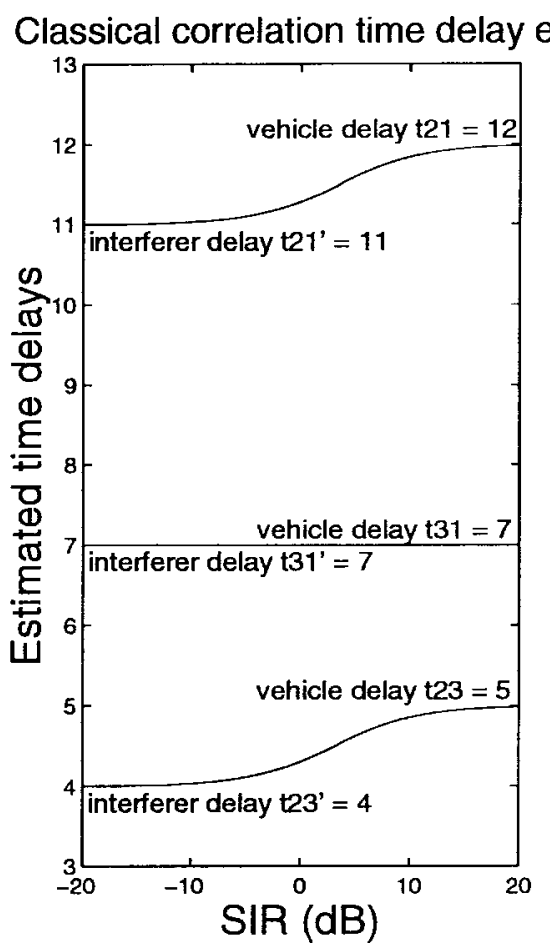

(b)

Fig. 9. Comparison of eigenvector and classical correlation methods on time delay estimations.

\section{Source Localization by LS Method}

The problem of source localization from arrival time delay estimates requires solving a set of nonlinear equations, which is computationally intractable. The computational problem is even worse if the propagation velocity is unknown. By introducing two auxiliary variables the solution is obtained from an over determined system of linear equations. The LS solution is not identical to the original source localization solution, but does yield an excellent approximation for seven or more sensors in a three-dimensional scenario (six or more sensors in a two-dimensional scenario). The LS solution of these linear equations can be found by traditional algebraic methods. 


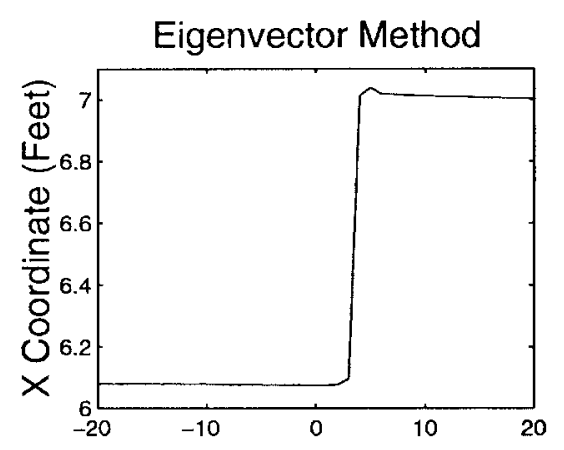

(a)

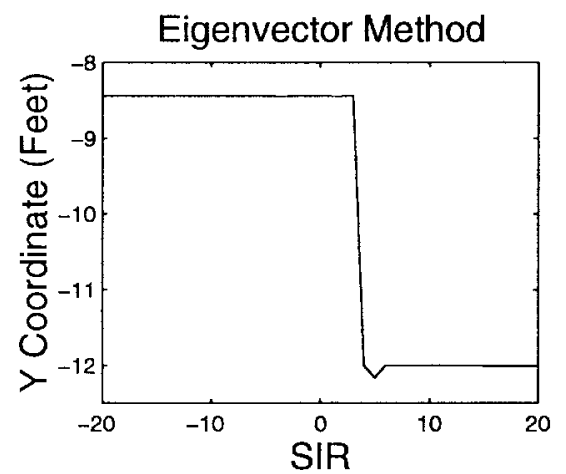

(c)

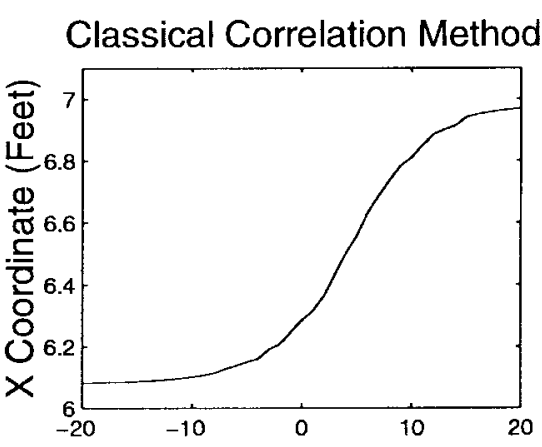

(b)

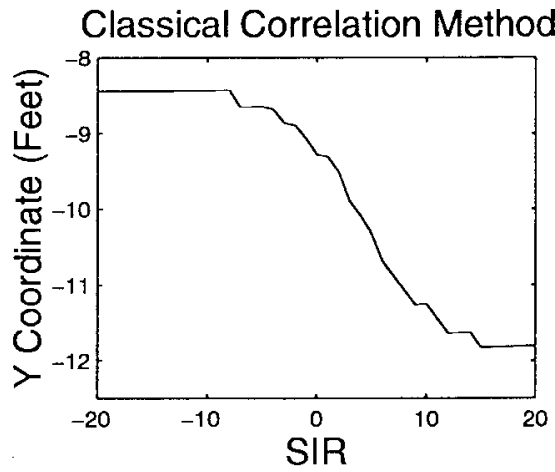

(d)

Fig. 10. Comparison of eigenvector and classical correlation methods on source location estimation.

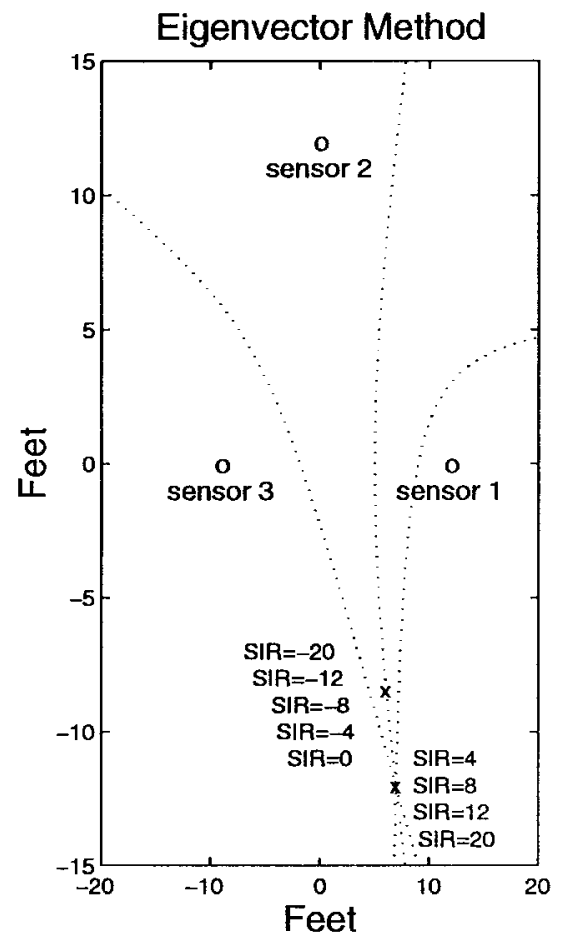

(a)

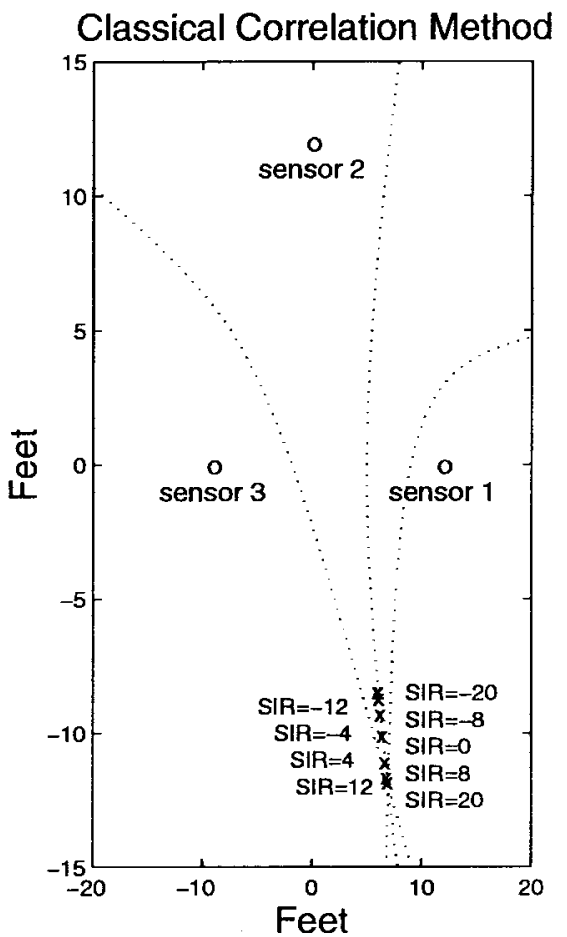

(b)

Fig. 11. Graphical interpretation of source localization based on time delay estimation for eigenvector/classical correlation methods.

The source localization problem in the random array context is to estimate a source location vector $\mathrm{r}_{s}$ given the $N$ sensor locations $r_{i}$ and the relative time delays, $t_{i}-t_{j}$ between the $i$ th sensor and a fixed $j$ th sensor. Without loss of generality, we choose $j=1$. The speed of propagation $v$ in this formulation can also be estimated from the data. In some problems $v$ may be considered to be partially known, while in others it is unknown. The relative time delays satisfy

$$
t_{i}-t_{1}=\frac{\left|\mathbf{r}_{i}-\mathbf{r}_{s}\right|-\left|\mathbf{r}_{1}-\mathbf{r}_{s}\right|}{v}, \quad i=2, \cdots, N .
$$

This set of $(N-1)$ equations in (12) involves the unknown 
TABLE II

SNR IMPROVEMENT USING BLIND BEAMFORMING

\begin{tabular}{lr}
\hline Condition & SNRI (dB) \\
1. True time delay beamformer & 10.0 \\
2. Eigenvector method with 4 time taps & 12.9 \\
3. Eigenvector method with 10 time taps & 16.1 \\
4. Eigenvector method with 19 time taps & 18.7 \\
5. Presteering-cigenvector method with 4 time taps & 13.5 \\
6. Presteering-eigenvector method with 10 time taps & 16.2 \\
\hline
\end{tabular}

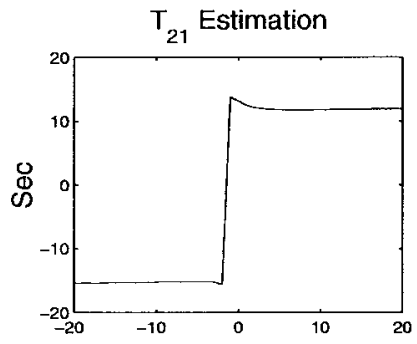

(a)

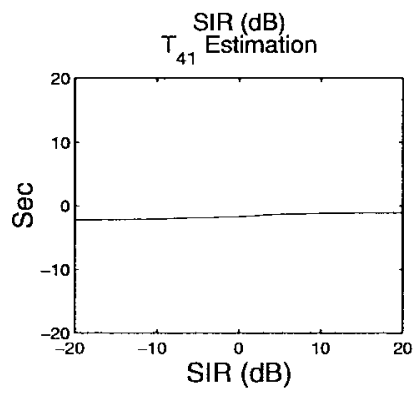

(c)

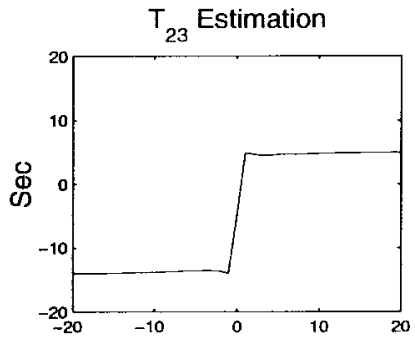

(b)

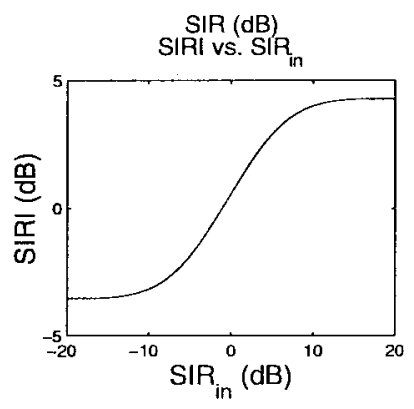

(d)
Fig. 12. Relative time delay estimations on $T_{21}, T_{23}$, and $T_{41}$ versus SIR and SIRI versus SIR $_{\text {in }}$ of two spectrally similar but spatially separated sources.

source position vector $r_{s}$ in a nonlinear manner, which makes finding its solution to be nontrivial.

Now, consider a reformulation of this problem. Equation (12) is equivalent to

$$
t_{i}-t_{1}+\frac{\left|\mathbf{r}_{s}-\mathbf{r}_{1}\right|}{v}=\frac{\left|\left(\mathbf{r}_{i}-\mathbf{r}_{1}\right)-\left(\mathbf{r}_{s}-\mathbf{r}_{1}\right)\right|}{v} .
$$

Upon squaring both sides, we have

$$
\begin{aligned}
\left(t_{i}-t_{1}\right)^{2}+ & 2\left(t_{i}-t_{1}\right) \frac{\left|\mathbf{r}_{s}-\mathbf{r}_{1}\right|}{v} \\
& =\left(\frac{\left|\mathbf{r}_{i}-\mathbf{r}_{1}\right|}{v}\right)^{2}-\frac{2\left(\mathbf{r}_{i}-\mathbf{r}_{1}\right) \cdot\left(\mathbf{r}_{s}-\mathbf{r}_{1}\right)}{v^{2}} .
\end{aligned}
$$

Algebraic manipulations of (14) yields

$$
\begin{array}{r}
-\frac{\left(\mathbf{r}_{i}-\mathbf{r}_{1}\right) \cdot\left(\mathbf{r}_{s}-\mathbf{r}_{1}\right)}{v\left|\mathbf{r}_{s}-\mathbf{r}_{1}\right|}+\frac{\left|\mathbf{r}_{i}-\mathbf{r}_{1}\right|^{2}}{2 v\left|\mathbf{r}_{s}-\mathbf{r}_{1}\right|}-\frac{v\left(t_{i}-t_{1}\right)^{2}}{2\left|\mathbf{r}_{s}-\mathbf{r}_{1}\right|} \\
=\left(t_{i}-t_{1}\right) .
\end{array}
$$

Now define the normalized position location vector variable and the two new auxiliary variables by

$$
\begin{aligned}
& \mathbf{x}_{\mathbf{s}} \equiv\left[x_{1}, x_{2}, x_{3}\right]^{T}=\frac{\mathbf{r}_{s}-\mathbf{r}_{1}}{v\left|\mathbf{r}_{s}-\mathbf{r}_{1}\right|} ; x_{4} \equiv \frac{1}{2 v\left|\mathbf{r}_{s}-\mathbf{r}_{1}\right|} ; \\
& x_{5} \equiv \frac{v}{2\left|\mathbf{r}_{s}-\mathbf{r}_{1}\right|} .
\end{aligned}
$$

Using the variables of (16), then (15) can be expressed as

$$
\begin{aligned}
& -\left(\mathbf{r}_{i}-\mathbf{r}_{1}\right) \cdot \mathbf{x}_{\mathbf{s}}+\left|\mathbf{r}_{i}-\mathbf{r}_{1}\right|^{2} x_{4}-\left(t_{i}-t_{1}\right)^{2} x_{5} \\
& \quad=\left(t_{i}-t_{1}\right), i=2, \cdots, N .
\end{aligned}
$$

Thus, (16) is a set of linear system of equations

$$
\mathbf{A x}=\mathbf{b}
$$

where

$$
\mathbf{A}=\left[\begin{array}{ccc}
-\left(\mathbf{r}_{2}-\mathbf{r}_{1}\right) & \left|\mathbf{r}_{2}-\mathbf{r}_{1}\right|^{2} & -\left(t_{2}-t_{1}\right)^{2} \\
\cdots & \cdots & \cdots \\
-\left(\mathbf{r}_{N}-\mathbf{r}_{1}\right) & \left|\mathbf{r}_{N}-\mathbf{r}_{1}\right|^{2} & -\left(t_{N}-t_{1}\right)^{2}
\end{array}\right]
$$

$\mathrm{x}=\left[\mathbf{x}_{\mathbf{s}} \mathbf{T}, x_{4}, x_{5}\right]^{T}$, and $\mathrm{b}=\left[t_{2}-t_{1}, \cdots, t_{N}-t_{1}\right]^{T}$. We note that in the three-dimensional problem, the first column on the right-hand side of (19) is a submatrix of dimension $(N-1) \times 3$ and thus $\mathbf{A}$ is an $(N-1) \times 5$ matrix, $\mathbf{x}$ is a $5 \times 1$ vector, and b is an $(N-1) \times 1$ vector. In the two-dimensional problem, the first column on the right-hand side of (19) is a submatrix of dimension $(N-1) \times 2$ and thus $\mathbf{A}$ is an $(N-1) \times 4$ matrix, $\mathbf{x}$ is a $4 \times 1$ vector, and $\mathrm{b}$ is an $(N-1) \times 1$ vector.

In the general three-dimensional case there are five unknowns in $\mathrm{x}$, but only four degrees of freedom in $v$ and $x, y$, and $z$ values of $\mathbf{r}_{s}$. To obtain an overdetermined solution we need at least five independent equations, which can be derived from the data of six sensors since the reference sensor does not generate an equation. Even placing six sensors randomly does not provide much assurance against ill-conditioned solutions. The preferred approach would be to use seven or more sensors, yielding six or more relative delays, and perform an LS fitting of the data. The minimum number of needed sensors can be reduced by one if the propagation velocity $v$ is known. In general, if $\left\{\hat{\mathbf{x}}_{\mathbf{s}}, \hat{x}_{4}, \hat{x}_{5}\right\}$ are the LS solution of (18), then the desired source location and the velocity are given by

$$
\hat{\mathbf{r}}_{s}=\frac{\hat{\mathbf{x}}_{s}}{2 \hat{x}_{4}}+\mathrm{r}_{1}, \quad \hat{v}=\sqrt{\frac{\hat{x}_{\tilde{z}}}{\hat{x}_{4}}} .
$$

If the source and sensors are known to be in the twodimensional space, then all the above results are valid except that the minimum number of sensors can be reduced by one.

While the above unified treatment is applicable to both the near-field and far-field cases, it is of interest to consider the far-field case when the distance to the source becomes large. The source angles are then the only parameters of interest. 


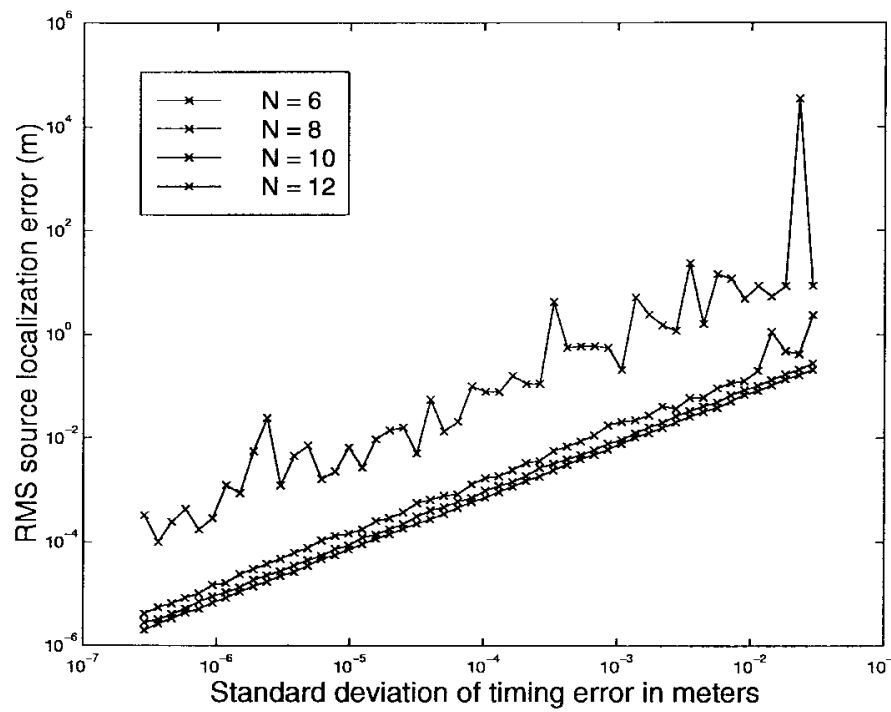

Fig. 13. RMS source localization estimation error versus time delay error.

The components of $\mathbf{u}=\left(\mathbf{r}_{s}-\mathbf{r}_{1}\right) /\left(\left|\mathbf{r}_{s}-\mathbf{r}_{1}\right|\right)$ are the direction cosines of the source angles and then $\hat{\mathbf{u}}=\hat{v} \hat{\mathbf{x}}$.

For the three-dimensional case, if the matrix $\mathbf{A}$ is well conditioned, and there are six sensors with no time delay estimation errors, then the LS solution of the source localization method will correctly identify the source locations. If, however, there is some timing error, or the matrix $\mathbf{A}$ is ill conditioned, the error may be substantial. For just six sensors, if the relative time delays are approximately equal, then $\mathbf{A}$ may be ill conditioned. As the number of sensors is increased, however, there is less likelihood of $\mathbf{A}$ being ill conditioned. It is also expected that the source localization errors are linearly related to the time delay estimation errors.

Computer simulations are used to investigate the performance of the source localization method. The simulations are based on the following model. A single source sends a signal to $N$ sensors. For each trial, both the source and the sensors are randomly located in a three-dimensional cube with each side being $10 \mathrm{~m}$. The sensors are independently and uniformly distributed in each of the three dimensions. The simulation assumes that the acoustic signals propagate in an ideal air channel with a speed of $v=343 \mathrm{~m} / \mathrm{s}$. In the solution to the problem, however, we assume this speed is unknown a priori. An uniformly distributed time delay estimation error is added to the relative time delays for each pair of sensors. The LS solution of the augmented linear equations is then used to estimate the location of the source and the speed of propagation, as discussed above. For each sensor and source location, 1000 iterations were performed using double-precision floating points.

The results for the estimation of the source location rootmean-square (rms) error are shown in Fig. 13 and the results for the propagation velocity rms error are shown in Fig. 14. These results show that the localization and velocity estimation errors are approximately linearly related to the delay estimation error. As expected, the error is reduced as the number of sensors is increased. Additional computations showed that the condition numbers essentially are independent of the timing error variance.

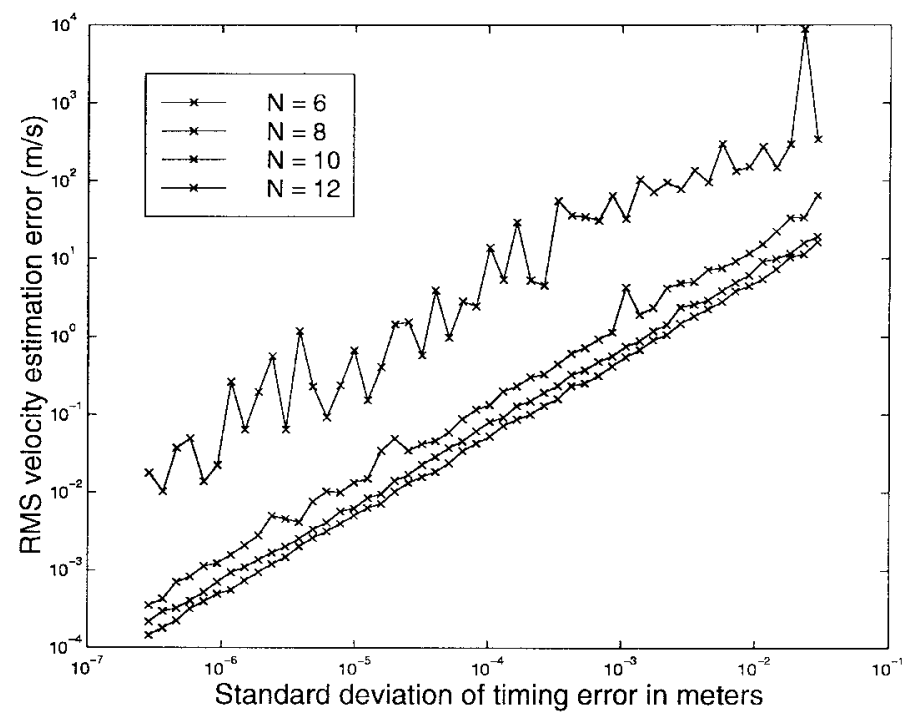

Fig. 14. RMS velocity estimation error versus time delay error.

\section{CONCLUSIONS}

In this paper we have considered blind beamforming for an array of randomly distributed sensors. In Section II, the ensemble averaged correlation matrix of the received sensor data vector was used to perform maximum power collection from the source with the highest peak power spectral density. The array weights were obtained from the dominant eigenvector of a matrix eigenvalue problem. We also showed that the ensemble averaged correlation matrix approach has a timeaveraged sample correlation matrix approach and an equivalent direct data matrix approach. Section III showed that the generalized Szegö theory of asymptotic distribution of eigenvalues of Toeplitz form can be used to explain the maximum power collection property of the array. Relative phase information among the weights of the array yielded relative time delays from the dominant source to the array sensors. In Section IV, various properties of blind beamforming time delay estimation and its applications to source localizations were considered. A general source localization scheme based on LS time delay estimation was presented in Section V.

In a practical sensor-array system, various types of imperfections limit the performance of the system. We are continuing to investigate degradations due to the signal quantization error, clock error due to imperfect time-synchronization among the sensors, and loss of coherence of the received signal among the sensors. Timing errors under consideration include synchronization offset, sample jitter, sample rate mismatch, and sample rate drift. If the clocks on different sensors are not perfectly synchronized, data samples will not be taken at exactly the same time. This offset will limit the performance of time delay estimation and can degrade the performance of a beamformer. Sample jitter occurs when the sample clock on a processor does not trigger at a constant rate. Instead, the sampling interval changes slightly from sample to sample. Sample rate mismatch occurs when the sensors are not clocked at exactly the same rate. Sample rate drift is the change in clock frequency over time. For systems in which the sensors are separated by large distance, 
environmental variations may cause the signal to lose complete coherence between channels. We are continuing to investigate modeling these errors and their effect on the performance of the proposed method. Another area of investigation is the use of presteered beamforming, based upon the use of blind beamforming time delay estimates derived through the dominant eigenvector method, as described in this paper. The integer part of the time delay estimates can be used to approximately delay steer the array so that a desired source appears to arrive at all sensors at the same time. Thus, there is low frequency domain distortion. Then adaptive beamforming with a small number of time taps can be used to further improve the SNR. An advantage of this is that the covariance matrix for this beamformer will be a subset of that computed to form the time delay estimates. Many adaptive beamforming algorithms can used, including the dominant eigenvector method described above. This method may provide good SINR improvement at the cost of low degradation for wideband signals.

\section{REFERENCES}

[1] J. K. Madisetti and W. B. Williams, The Signal Processing Handbook. Boca Raton, FL: CRC, 1998.

[2] R. O. Schmidt, "Multiple emitter location and signal parameter estimation," IEEE Trans. Antennas Propagat., vol. 34, pp. 276-280, 1986.

[3] R. Roy, A. Paulraj, and T. Kailath, "ESPRIT - a subspace rotation approach to estimation of parameters of cisoids in noise," IEEE Trans. Acoustics, Speech, Signal Processing, vol. 34, pp. 1340-1342, 1986.

[4] Y. Bresler, V. U. Reddy, and T. Kailath, "Optimum beamforming for coherent signals and interferences," IEEE Trans. Acoustics, Speech, Signal Processing, vol. 36, pp. 883-843, 1988.

[5] P. Stoica, M. Viberg, K. M. Wong, and Q. Wu, "Maximum-likelihood bearing estimation with partly calibrated arrays in spatially correlated noise," IEEE Trans. Signal Processing, vol. 44, pp. 888-899, 1996.

[6] H. Wang and M. Kaveh, "Coherent signal subspace processing for the detection and estimation of angles of arrival of multiple wideband sources," IEEE Trans. Acoustics, Speech, Signal Processing, vol. 3, pp. 823-831, 1985

[7] D. T. M. Slock, "Blind fractionally-spaced equalization, perfect reconstruction filter banks and multichannel linear prediction," in Proc. IEEE ICASSP, 1994, pp. 585-588.

[8] B. G. Agee, S. V. Schell, and W. A. Gardner, "Spectral self-coherence restoral: A new approach to blind adaptive signal extraction using antenna arrays," Proc. IEEE, vol. 78, pp. 753-767, 1990.

[9] R. P. Gooch and J. Lundell, "The CM array: an adaptive beamformer for constant modulus signals," in Proc. IEEE ICASSP, 1986, pp. 2523-2526.

[10] A. J. vanderVeen and A. Paulraj, "An analytical constant modulus algorithm," IEEE Trans. Signal Processing, vol. 44, pp. 1136-1155, 1996.

[11] A. Souloumiac, "Blind source detection and separation using second order nonstationarity," in Proc. IEEE ICASSP, 1995, pp. 1912-1915.

[12] M. C. Doğan and J. M. Mendel, "Cumulant-based blind optimum beamforming," IEEE Trans. Aerosp. Electron. Syst., vol. 30, pp. 722-740, 1994

[13] F. Lorenzelli and K. Yao, "Arrays of randomly spaced sensors," Proc. SPIE, vol. 2846, pp. 122-133, 1996.

[14] J. C. Nash. Compact Numerical Methods for Computers. New York: Wiley, 1979.

[15] G. H. Golub and C. F. Van Loano, Matrix Computations, 2nd ed. Baltimore, MD: Johns Hopkins Press, 1989.

[16] U. Grenander and G. Szegö, Toeplitz Forms and Their Applications. Berkeley CA: Univ. of California Press, 1958.

[17] R. E. Blahut, Principles and Practice of Information Theory. Menlo Park, CA: Addison Wesley, 1987.

[18] T. Koga and F. Cheng, "Extension of the Toeplitz theorem and its application," in Proc. GLOBECOM 1987, 1987, vol. 1, pp. 263-267.

[19] P. A. Voois, "A theorem of the asymptotic eigenvalue distribution of Toeplitz-block-Toeplitz matrices," IEEE Trans. Signal Processing, vol. 44, pp. 1833-1841, 1996.

[20] T. Pham, ARL, private communication, Sept. 1997.

[21] G. C. Carter. Coherence and Time Delay Estimation. New York: IEEE, 1993.

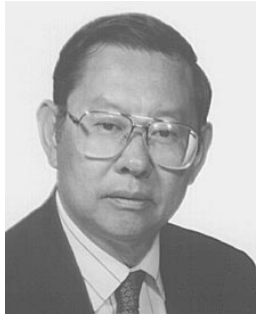

Kung Yao (S'59-M'65-SM'91-F'94) received the B.S.E. (highest honors), M.A., and Ph.D. degrees in electrical engineering from Princeton University, Princeton, $\mathrm{NJ}$

$\mathrm{He}$ has worked at Princeton-Penn Accelerator, the Brookhaven National Laboratory and Bell Telephone Laboratories, Murray Hill, NJ. He was an NAS-NRC Post-Doctoral Research Fellow at the University of California, Berkeley. In 1969 he was a Visiting Assistant Professor at the Massachusetts Institute of Technology, Cambridge, MA. From 1973 to 1974 he was a Visiting Associate Professor at the Eindhoven Technical University, Eindhoven, the Netherlands. From 1985 to 1988 he served as an Assistant Dean of the School of Engineering and Applied Science at the University of California at Los Angeles (UCLA). He is currently a Professor in the Electrical Engineering Department at UCLA. His research and professional interests include systolic and VLSI algorithms, architectures and systems, digital and acoustic signal processing, microphone and sensor array processing, chaotic communication, digital communication theory, radar system, and simulation. He has published more than 200 journal and conference papers.

Dr. Yao received the IEEE Signal Processing Society's 1993 Senior Award in VLSI Signal Processing. He is the coeditor of the two volume series High Performance VLSI Signal Processing (New York: IEEE, 1997). He has served as Program Chair, Secretary, and Chair of the IEEE Information Theory Group and served two terms as a member of the Board of Governors of the IEEE Information Theory Group. He was a Cochair of the 1981 International Symposium on Information Theory and the Representative of the IT-BOG of the 1987 IEEE Information Theory Workshop. He is a member of the VLSI Technical Committee of the IEEE Signal Processing Society. He was also the Chair of the Technical Program and the General Chair of the 1990 and 1992 IEEE Workshop on VLSI Signal Processing. He has served as an Associate Editor for Book Reviews of the IEEE TRANSACTIONS. ON INFORMATION THEORY and was an Associate Editor of Probability in the Engineering and Informational Sciences. In 1991-1993, he was an Associate Editor for VLSI Signal Processing of the IEEE TRANSACTION ON CIRCUITS AND SYSTEMS. He is a member of the Editorial Board of the Journal of VLSI Signal Processing and Integration.

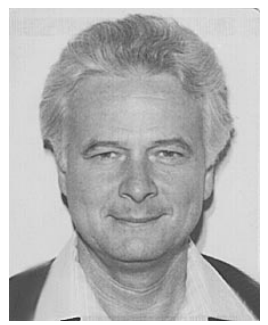

Ralph E. Hudson received the B.S. degree from the University of California at Berkeley in 1960 and the $\mathrm{Ph} . \mathrm{D}$. degree from the U.S. Naval Postgraduate School, Monterey, CA, in 1969.

$\mathrm{He}$ is a Research Engineer in the Electrical Engineering Department at the University of California at Los Angeles. His research interests include synthetic array radar, modern portfolio theory, and signal and array processing.

Dr. Hudson received the Legion of Merit and Air Medal, while serving in the U.S. Navy, and the Hyland Patent Award in 1992, while working as Chief Scientist at Hughes Aircraft Company.

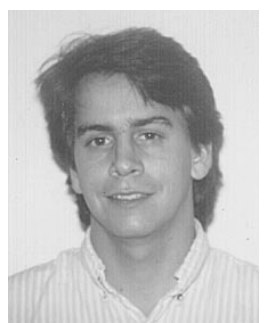

Chris W. Reed received the B.S. and M.S. degrees in electronic engineering from California Polytechnic State University at San Luis Obispo, in 1993 and 1994, respectively.

He served in the U.S. Army from 1985 to 1989 From 1990 to 1991 he was with Compaq Computer, Houston, TX, and in 1993 he worked for the Applied Research Facility, San Luis Obispo, CA. Since 1994, he has been with Raytheon Systems Company (formerly Hughes Aircraft) in El Segundo, CA, where he is a Staff Engineer in the Advanced and Adaptive Processing Signal Processing Systems Department of the Sensors and Electronic Systems group. He is currently a Raytheon Fellow pursuing the $\mathrm{Ph} . \mathrm{D}$. degree in electrical engineering at the University of California at Los Angeles. His research interests include source localization adaptive beamforming, estimation theory, and numerical linear algebra. 


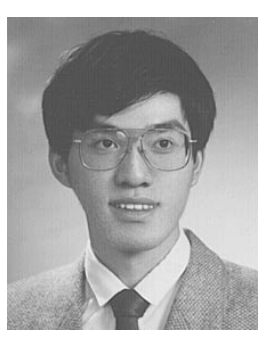

Daching Chen was born in Taipei, Taiwan, in 1970. $\mathrm{He}$ received the B.S.E.E. degree from National Taiwan University, Taipei, Taiwan, in 1992 and the M.S.E.E. and Engineering degrees from the University of California, Los Angeles, in 1995 and 1996, respectively. He is currently working toward the Ph.D. degree at the Department of Electrical Engineering, the University of California, at Los Angeles.

From 1992 to 1994 he served in the Taiwanese Army as a Second Lieutenant. In 1996, he joined the CDMA development group of VLSI Technology Inc., Irvine, CA, where he works as a Senior Design Engineer His current research interests include sensor array processing, adaptive filtering theory, and advanced signal processing techniques for wireless communications.

Flavio Lorenzelli graduated cum laude from Politecnico de Torino, Italy, in 1986. From 1989 to 1993 he was a Fulbright scholar at the University of California at Los Angeles (UCLA). He received the M.S. and Ph.D. degrees in electrical engineering from UCLA in 1993.

From 1986 to 1988 he was a Research Engineer at the CSELT Telecommunications Laboratories, Torino, Italy. From 1993 to 1996 he was part of the research staff at the Electrical Engineering Department, UCLA. Since 1996 he has been with SGS-Thomson, Milan, Italy. His research interests include systolic array design, algorithms for signal processing, array processing with radar and acoustic applications, multimedia, and computer architecture. 